\title{
Study of the destructive activity of microorganisms isolated from soil contaminated by pesticides
}

\author{
Azhar Malik ${ }^{1,2, *}$, Gulzhamal Abdieva ${ }^{1,2}$, Perizat Ualieva ${ }^{1,2}$, and Nuraly Akimbekov ${ }^{1,2}$ \\ ${ }^{1}$ Al-Farabi Kazakh National University, Faculty of biology and biotechnology, Biotechnology Department, 71 al-Farabi Ave., Almaty, \\ Republic of Kazakhstan \\ ${ }^{2}$ Scientific Research Institute of Biology and Biotechnology Problems, 71 al-Farabi Ave., Almaty, Republic of Kazakhstan
}

\begin{abstract}
Chemical pollution of natural and agricultural habitats is a growing global problem and a serious threat to the sustainability and health of people. Among various chemical ecotoxicants of anthropogenic origin, organochlorine pesticides are among the most stable and dangerous for the environment and humans. Pesticides are chemical plant protection products, the intensive and not always justified the use of which has led to the fact that recently they are also considered among the most dangerous pollutants. One of the serious environmental problems is the pollution of natural objects with organic pesticides with high toxicity and persistence. Toxic substances can enter the environment and pose a threat to all living organisms, including the microbial population of the soil. Microorganisms isolated from ecosystems exposed to longterm treatment with pesticides can quickly decompose these compounds. The problem related to the decomposition of pesticide residues in the soil is an important environmental issue. Microorganisms are able to most effectively decompose xenobiotic substances foreign to the biosphere. The ability of microorganisms to decompose pesticides is associated with biochemical reactions and a high level of their adaptation. In this study, the microbial diversity of the soil in the territories adjacent to the pesticide burial sites was studied.
\end{abstract}

\section{Introduction}

At present, there is an acute environmental problem related to the effects of many years of use in agriculture of pesticides, chemical agents for controlling pests of agricultural plants. Pesticides, which are composed of insecticides, herbicides, and fungicides, are a group of potentially toxic substances that can disrupt the microbial structure and function in habitats.

As it is known, at present, their use is practically prohibited, but due to their high toxicity with respect to soil bio-objects and water bodies, another problem has arisen, the leveling of which is connected with the need to create new environmentally hazardous objects storages (warehouses) for huge amounts of unused pesticides.

Considering the above, special storage facilities in a number of regions were built for pesticides available in warehouses. In particular, they are in the Talgar district, Almaty region.

Although, all the studies conducted earlier in this direction was devoted to study the target activity of pesticides in the process of their application in agriculture. Nowadays the attention of researchers is also related to the problems associated with the influence of pesticide storage facilities on the environment, on the physiological activity of microorganisms and other living objects of water and soil, which can serve as markers of change, based on the presence of pesticides and their decomposition products in environmental objects. Pesticides can have a direct toxic or beneficial effect on microbial communities, similar to those reported for higher organisms. Some microbes, in particular, bacteria, can use pesticides as a source of nutrients that promote their growth and survival, while sensitive species can be damaged or destroyed by pesticides [1].

It is possible to assess the intensity and nature of the effects of toxic substances by their metabolic activity, such as pesticides, on metabolic processes in the cells of soil microorganisms directly, i.e. metabolic potential of soil microbial communities in pesticide burial areas.

It is equally important that the study of the microbial communities of soils contaminated with pesticides is necessary both for the assessment of biological risk and for the selection of promising agents for bioremediation activities in contaminated areas. The method of cleaning soils contaminated with pesticides using microorganisms-biodestructors is undoubtedly effective and cost- effective [2].

In connection with the above purpose, further research was the study of the microbial diversity of the soil from the pesticides burial site, the screening of effective destructive microorganisms and the study of the destructive potential of crops [3].

\footnotetext{
Corresponding author: azhar.malikkyzy@gmail.com
} 
We have conducted studies on the microbial diversity of soil samples in Almaty region (Fig.1), adjacent to the burial places of pesticides (test samples) and background areas (control samples).

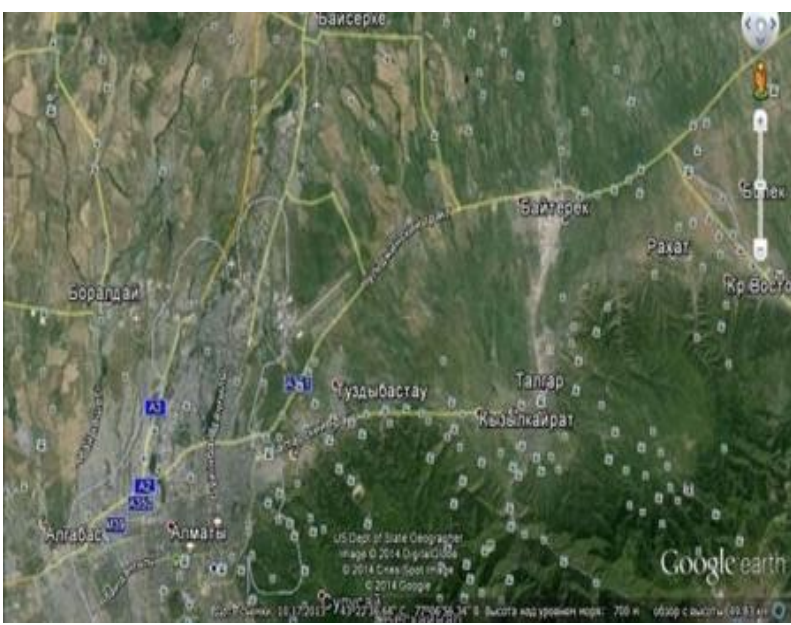

Fig.1. Soil sampling points in Almaty region

\section{Materials and methods}

Determination of the destructive activity of microorganisms

The search for destructors was carried out among cultures from the dominant populations of microorganisms. Indication of the destructive strains in the composition of the total soil microbiota was carried out on solid medium M9 with the addition of a pesticide as a carbon source $0.01 \%, 2,3,5$-triphenyl tetrazolium chloride (TTCH) as an indicator of dehydrogenase activity of bacteria [4]. The destructive activity of the cultures was evaluated by the activity of growth and preservation of cell viability in the presence of organochlorine compounds [5].

The use of a wide range of pesticides suggests different mechanisms of action of these substances on prokaryotic and eukaryotic cells of microorganisms, on heterotrophic microorganisms, and the spectrum of these mechanisms is very wide. Therefore, the strains of isolated pure cultures showed different growth activities in the medium with the organochlorine preparation [6].

Sequencing

Extraction and PCR amplification of genomic DNA The genomic DNA sample was extracted by SDS, and then by agarose gel electrophoresis, which determines the purity and the concentration of DNA. An appropriate amount of sample is taken in a centrifuge tube and the sample is diluted to $1 \mathrm{ng} / \mathrm{ml}$ of sterile water. Using low genomic DNA as a template, PCR was used to provide amplification based on the selection of selection methods using specific barcode primers, master mix for highperformance Phusion ${ }^{\circledR}$ PCR with GC buffer from New England Biolab and high-precision enzymes. Efficiency and accuracy.

Primer related area: Primers 16S V4-region (515F and 806R): identification of bacterial diversity; Primers 18S V4-region (528F and 706R): identification of eukaryotic microbial diversity; Primers for the ITS1 region (ITS5-
$1737 \mathrm{~F}$ and ITS2-2043R): identification of fungal diversity; In addition, the amplified region also includes 16S V3-V4 / 16S V4-V5; Archaea 16S V4; 18S V9 and ITS2.

Mixing and cleaning PCR products

The PCR product was determined by electrophoresis using a $2 \%$ agarose gel.

Samples were mixed according to the concentration of the PCR product. After thorough mixing, the PCR product was purified by electrophoresis using $1 \times \mathrm{TAE}$ concentration of $2 \%$ agarose gel. Group. The product cleaning kit uses the Thermo Scientific GeneJET glue regeneration kit to restore the product.

Library creation and sequencing

The library was constructed using the Rxns Kit 48 library of Thermofisher Ion Plus library fragments. The created library was quantified by Qubit and tested by the library, and then sequenced using Life Ion S5 TM from Thermofisher or Ion S5 TM XL.

\section{Results and discussion}

The screening of active microorganisms-destructors of persistent organic pollutants and their decomposition products was performed on all strains of pure cultures isolated from the soil adjacent to the pesticides burial sites. Strains K3, AK5 isolated from the burial site of pesticides have high destructive activity against pesticides. The results are presented in Fig. 2.

As can be seen from Figure 2, the number of cells of the studied strains after 24 hours was $6,4 \times 10^{4}$ for K3, while for AK5 - 1,3 x $10^{5} \mathrm{CFU} / \mathrm{g}$. On the fifth day (120 hours) the growth of the cultures $\mathrm{K} 3$ decreased to $5,6 \mathrm{x}$ $10^{4}$, while the number of cells of AK5 reached 2,5 $\mathrm{x}$ $10^{5} \mathrm{CFU} / \mathrm{g}$.

As can be seen, from Figure 3, the strain of cultures of microorganisms-destructors on the medium with the addition of pesticide form surface colonies of 1-2.5 mm in size. When growing crops on M9 medium with pesticides, the colonies had a larger size $-1.8-3 \mathrm{~mm}$. The colonies were round, with a smooth edge and a smooth surface, matt red. The structure of the colonies is homogeneous. Almost all strains formed S-type colonies, sometimes mucous (O) colonies after surface sowing. Strains K3, AK5 were observed round and smooth, shiny colonies. On M9 medium with pesticides, all strains formed different zones of enlightenment around the colonies.

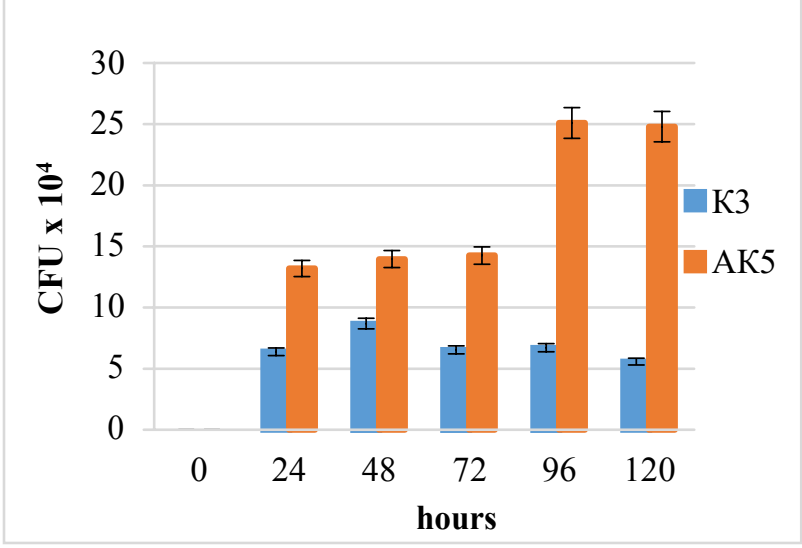


Fig.2. Growth of the culture medium with the addition of pesticides as the sole carbon source.

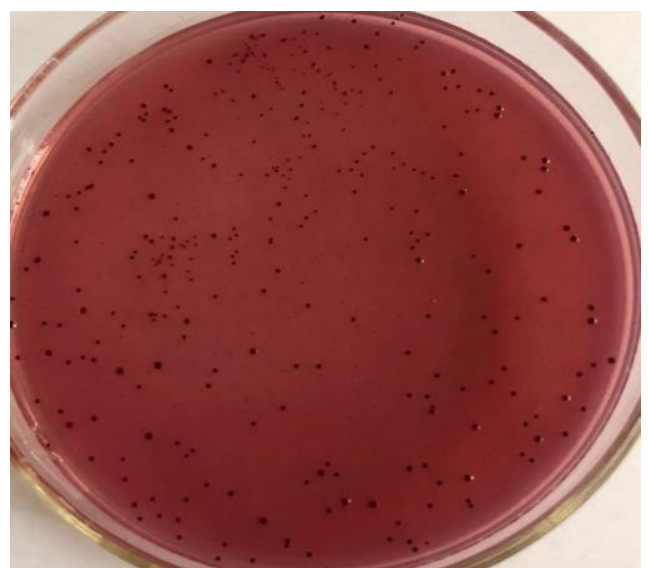

Fig. 3. The growth of cultures of microorganisms-destructors on the medium with the addition of pesticide

It should be noted that on a solid medium M9 all colonies were only smooth, shiny S-forms of red color. Cultures on M9 medium with addition of pesticide, as the only carbon source, change the initial white-matte color to red, which is due to the formation of reduced triphenyl formazane (TFF) in the medium. The scientific literature provides numerous examples of the transformation of various pesticides under the action of microorganisms in certain conditions and certain soils [7]. Organochlorine preparations under the action of microflora are subjected to deep decomposition with splitting of aromatic rings.

A study of the chemical contaminant destructor strains revealed that the K3 and AK5 bacterial strains are capable of destroying the preparation, which was indicated by the coloration of the colonies and the environment around them to become red, indicating the formation of reduced TFF.

Since under aerobic conditions the first stage of biodegradation of xenobiotics is oxidative metabolism reactions catalyzed by various oxidoreductases, the main of which are dehydrogenases, the identification of these enzymes in microorganisms indicates the destructive potential of the culture. Molecular genetic identification of microorganisms was performed by Sanger sequencing. A 16S rRNA gene fragment of about 700 bp was amplified by PCR. As a result of molecular genetic identification, selected strains of microorganisms were identified: strain $\mathrm{K} 3$ was assigned to the species Bacillus aryabhattai, strain AK5 to the species Bacillus subtillis.

It is known that soil fertility and self-purification directly depend on the activity of microbiological processes, however, as a result of high soil intoxication, the autochthonous microflora is inhibited [8]. Therefore, the development of complex technologies aimed at restoring the basic functions of the soil and increasing their fertility is of considerable scientific interest, both for theoretical and for applied microbiology [9-10]. Currently, biological remediation methods are considered as priorities for solving the problem of cleaning contaminated soils.

To date, research in the field of molecular ecology of microorganisms is united under the common name metagenomic studies. The object of the study of metagenomics is a metagen, i.e. genetic material obtained directly from the environment without prior cultivation of microorganisms. $[11,12,13]$.

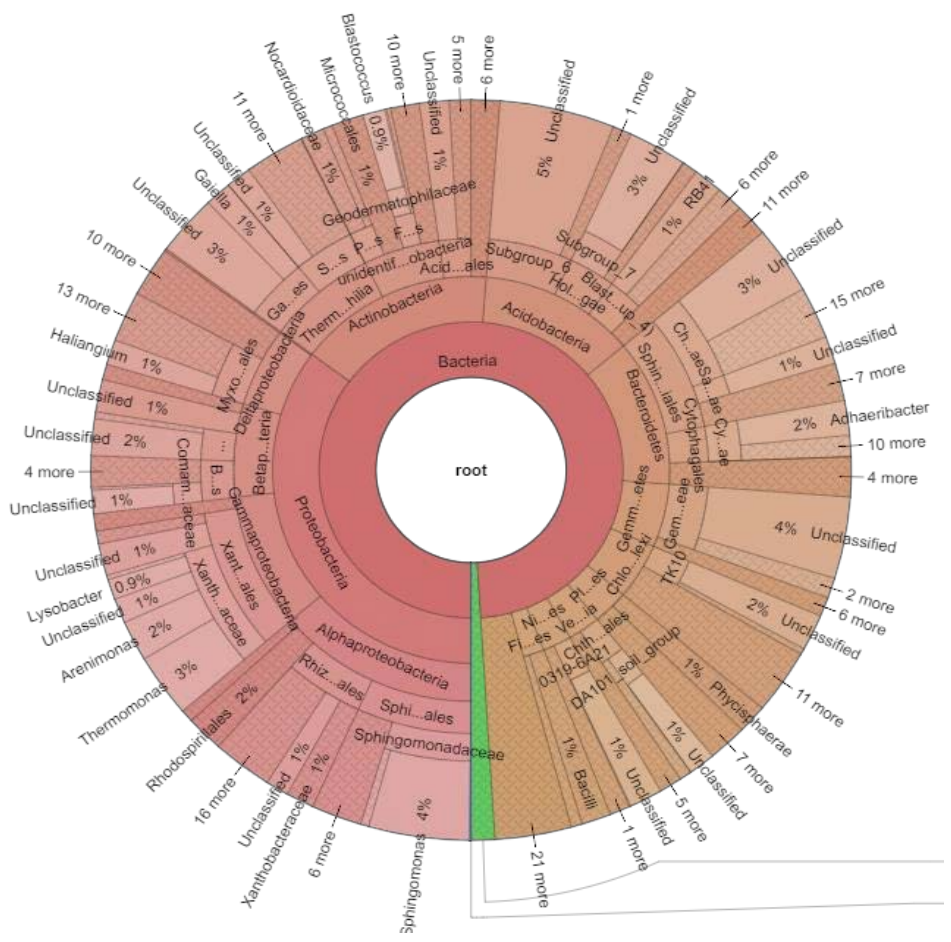

Fig. 4. The Krona graph of the relative abundance of annotated taxa in the Kyzylkairat. 
Figure 4 presents the results of the study of the total microflora of Kyzylkairat soil samples using metagenomic analysis of Illumina high-throughput sequencing. As a result of metagenomic analysis, the following physiological groups of microorganisms dominate in soil samples contaminated with pesticides: Proteobacteria 36\%, Actinobacteria 16\%, Acidobacteria $13 \%$, Bacteroidetes $12 \%$.

One of the urgent tasks of modern biotechnology is the creation of biologics based on destructive strains

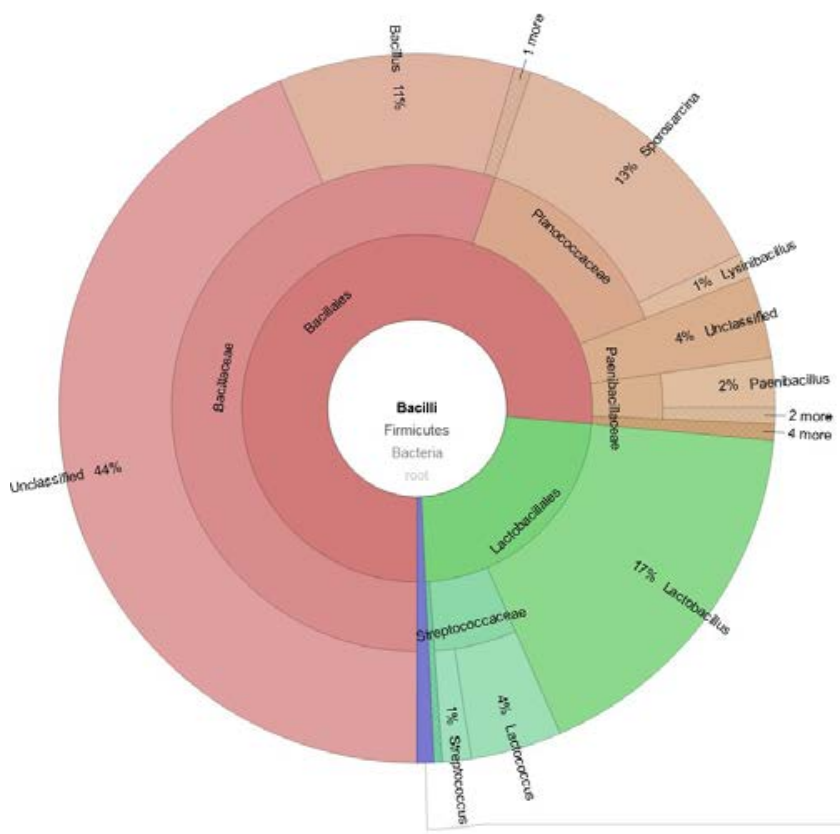

isolated from native microflora to solve a complex of tasks related to the rehabilitation of soils contaminated with xenobiotics.

Fig. 5. Krona taxonomy web visualization illustrating the share of bacteria of the genus Bacillus

In the study, as a result of studying the destructive activity of strains isolated from contaminated soil samples from Amangeldi №1 and Kyzylkairat, 2 strains were selected that have higher destructive activity against persistent organic pollutants. Additionally, molecular genetic identification results demonstrated that selected cultures of microorganisms are assigned to the genus Bacillus.

Figure 5 shows the visualization of the crown web taxonomy using the example of Bacillus. The composition of the microbial diversity of soil samples is dominated by spore-forming gram-positive bacteria of the Bacillaceae family of $72 \%$. In the microbiota of Kyzylkairat soil samples, the share of bacteria of the genus Bacillus was 14\%. These indicators indicate that the destructor dominates in the microflora of the polluted soil - organochlorines of organochlorine pesticides of the genus Bacillus.

\section{Conclusions}

Thus, the conducted studies indicate the need for continuous monitoring of the state of microbial diversity in the territory of adjacent to the pesticides burial sites, as well as studying the possibility of using microorganisms as indicators of environmental pollution. The search and selection of promising cultures of microorganisms capable of active degradation of persistent organic pollutants is the basis for the development of bioremediation measures to clean the soil from residual pesticides. The present study, on the one hand will provide reliable information not only on the impact of pesticides on the environmental situation of the investigated region, but also have predictive value, as they allow to judge about possible changes of the microbial potential in the presence of pesticides. Of equal important is the fact that the study of the microbial communities of soils contaminated with pesticides is necessary both for the assessment of biological risk and for the selection of promising agents for remediation in contaminated areas.

\section{References}

1. T. Doolotkeldieva, M. Konurbaeva, and S. Bobusheva, Environ Sci Pollut Res Int. 25, 31848 (2018)

2. T.P. Baczynski, D. Pleissner, T. Grotenhuis. Chemosphere, 78, 22 ( 2010)

3. R. Yusiasih, M. Efendi, M. Pitoi, H.R. Hariyadi. Earth and Environmental Science 160, 012023 (2018).

4. E. V. Vasnetsova, O. Yu. Ksenofontova, D. A. Tikhonova, E. A. Filimonova, K. V. Savina, Izv. 
Sarat. un-t. New ser. Ser. Chemistry. Biology. 16, 3 (2016)

5. E.N. Kulikova-Khlebnikova, Yu.V. Robertus, A.V. Kivatskaya, R.V. Lyubimov. Bull. of the Altai State Agr. Univ. 106, 59-63 (2013)

6. A.V. Kolupaev. Abs. of thesis. work. 44, 18-30 (2010)

7. Ye.V. Vasnetsova, O. Yu. Ksenofontova, D. A. Tikhonova, Ye. A. Filimonova, K. V. Savina. New sir Ser. Chemistry. 16, 3 (2016)

8. M. Geetha, M.H. Fulekar, Afr J Environ Sci Technol. 2, (2008)
9. M. Hayatsu, M. Hirano, S. Tokuda, Appl Environ Microbiol, 66, 1737 (2000)

10. F. Kafilzadeh, M. Ebrahimnezhad, Y. Tahery, Iran. Osong Public Health Res Perspect, 6, 39 (2015)

11. K. Yu, T. Zhang, PLoS ONE, 7 (2012)

12. M. Wexler, A. W. Johnston, Methods Mol Biol 668, 77 (2010)

13. L. P. Wackett, Envir. Micro. Wiley Online Library 14, 3066 (2012) 